\title{
The Role of Angle in the Evaluation of Ablation Accuracy in Pulmonary Vein Isolation Navigated by Image Integration
}

\author{
Song-Wen Chen, ${ }^{1}$ Li-Dong Cai, ${ }^{1}$ Tetsuya Asakawa, ${ }^{2,3}$ Gen-Qing Zhou, ${ }^{1}$ Yong Wei, ${ }^{1}$ Bao-Zhen Qi, ${ }^{1}$ \\ Zhi-Yu Ling, ${ }^{1}$ Hai-Qing Wu, ${ }^{1}$ Juan $\mathrm{Xu},{ }^{1}$ Shao-Wen Liu ${ }^{1}$ \\ ${ }^{1}$ Department of Cardiology, Shanghai General Hospital, Shanghai Jiao Tong University School of Medicine, Shanghai, China; \\ ${ }^{2}$ Department of Neurosurgery, Hamamatsu University School of Medicine, Higashi-ku, Hamamatsu-city, Shizuoka, Japan; \\ ${ }^{3}$ Research Base of Traditional Chinese Medicine Syndrome, Fujian University of Traditional Chinese Medicine, Fuzhou, China
}

\section{ABSTRACT}

Background: The conventional index for ablation accuracy is to compare the distance between mapping points with and without treatment by using image integration. We attempted to quantitatively evaluate the role of angle as an index in the ablation accuracy in patients with atrial fibrillation (AF).

Methods: A total of 48 patients with AF were included in the present study. Virtual fluoroscopy planes were predicted by pulmonary vein (PV) angiography, and the standard image planes were defined on the basis of the computed tomography images. Ablations were performed, guided by image integration; and the ablation planes were defined by the actual ablation rings. The predicted angle (distance) was defined as the angle (distance) between the fluoroscopy (predicted) plane and image (standard) plane, whereas the actual angle (distance) was defined as the angle (distance) between the ablation (actual) planes and the image (standard) planes.

Results: We found that all actual angles were significantly smaller than the predicted angles $(P<.05)$, but only the actual distances in the left $\mathrm{PV}$, right inferior $\mathrm{PV}$, right superior $\mathrm{PV}$, and right $\mathrm{PV}$ were significantly smaller; the distances in the left inferior PV and left superior PV were not significantly different $(P>.05)$.

Conclusion: Our finding indicates that both the angle and the distance can be significantly reduced by navigation with image integration, but that the angle exhibited better sensitivity than the conventional index of distance. We suggest that the angle should be considered as a new index for ablation accuracy.

Received October 3, 2018; accepted October 10,2018.

Song-Wen Chen and Li-Dong Cai are equal contributors.

Correspondence: Tetsuya Asakawa, MD, PhD, Department of Neurosurgery, Hamamatsu University School of Medicine, Handayama, 1-20-1, Higashiku, Hamamatsu-city, Shizuoka, 431-3192, Japan; +81-53-435-2283; fax: +81-53-435-2282 (e-mail: asakawat1971@gmail.com). Shao-Wen Liu, MD, PhD, FHRS, Department of Cardiology, Shanghai General Hospital, Shanghai Fiao Tong University School of Medicine, No. 100, Haining Road, Shanghai 200080, China; +86-21-63240090 ext. 3052; fax: +86-21-63240090 (e-mail: shaowen.liu@hotmail.com).

\section{INTRODUCTION}

The most widely used ablation processes for atrial fibrillation (AF) entail integrating circumferential pulmonary vein (PV) ablation and isolation. Technologies such as a local electrogram, an image integration system comprised of a threedimensional (3D) electroanatomic mapping system, and a single circular mapping catheter are usually employed to improve the ablation accuracy. It was emphasised in an expert consensus statement that care should be taken to identify the PV ostia to avoid ablation within the PV and lessen the risk for PV stenosis [Calkins 2012]. Therefore, correct identification of the PV ostium is crucial, with benefits by using technologies such as image integration.

Current image integration techniques (eg, CARTOMerge, Biosense Webster, Diamond Bar, CA, USA) incorporate computed tomography (CT) imaging or magnetic resonance imaging (MRI) into the 3D mapping system and are widely used in clinical practice [Kistler 2006; Desjardins 2016]. Although most previous studies have concluded that image integration has a positive influence on clinical outcomes [Marai 2016; Shigenaga 2016] and although other important parameters have been identified [Bertaglia 2009], the most commonly used index for evaluating the accuracy of ablation is comparison of the actual distances (between the ablation and image planes, guided by image integration) with the predicted distances (between the fluoroscopy and image planes, not guided by image integration) [Kimura 2013]. This distance is well documented as a suitable index for evaluating ablation accuracy.

In a previous study, we showed that there was a significant torsion angle between ipsilateral PVs and suggested that this should be considered during procedure planning to avoid single-plane circumferential ablation. In that study, we defined the torsion angle as "the two dihedral angles between the common ostium plane and the best ostium plane of the superior and inferior PVs" [Chen 2013]. When considering the torsion angle, we also believe the angles of the PV planes during ablation are important. As with a smaller distance, we hypothesize that a smaller angle is related to better accuracy and that the angle can be reduced by image integration.

In this study, we performed quantitative evaluation of the difference between angles measured with and without image integration. We aimed to verify whether the angle could be 
reduced by image integration and could be a potential index of ablation accuracy.

\section{MATERIALS AND METHODS}

\section{Subjects}

We enrolled 48 patients with $\mathrm{AF}$ in this study. For inclusion, patients were required to meet the following criteria: having symptomatic paroxysmal or persistent $\mathrm{AF}$, being scheduled for a first ever AF ablation guided by CARTO-Merge, and having a typical PV pattern consisting of 4 discrete PVs with 4 separate ostia. The pattern comprised the following: right superior PV (RSPV), left superior PV (LSPV), right inferior PV (RIPV), and left inferior PV (LIPV) [Mansour 2004]. The present study was performed in accordance with the Declaration of Helsinki (2000), and it was approved and supervised by the Ethics Committee of Shanghai Jiao Tong University (permission no.: 015724910308). All patients and relatives were given full explanations of all the procedures, and informed consent was obtained from the patients or their relatives.

\section{Ablation Setting}

We used the same preoperative preparation, imaging acquisition, and $\mathrm{AF}$ ablation procedures described in our previous studies [Chen 2012, Chen 2013]. Briefly, CT angiography of the PV was performed within 3 days before ablation, and the enhanced cardiac CT image was imported into the CARTO workstation; in the present study, a segmentation process was employed to obtain the structures of interest (left atrium [LA] and PVs) [Tops 2005].

\section{Definition of the Fluoroscopy Plane}

Before ablation, we defined the predicted planes for each patient, by using fluoroscopy. These predicted planes were called the "fluoroscopy planes." The fluoroscopy planes of the 4 separate PVs and 2 ipsilateral PVs were mapped and labelled under the guidance of contrast PV angiography, fluoroscopy, and the 3D mapping system, but without CARTOMerge, prior to $3 \mathrm{D}$ anatomic reconstruction of the LA. The fluoroscopy planes of the 4 separate PVs were defined by the anterior, posterior, and superior or inferior PV-LA junctions, and the 2 ipsilateral PVs were defined by the superior, inferior, and anterior or posterior PV-LA junctions. These points were marked in the CARTO system as FLS-1 to FLS-3, FL-1 to FL-3, and so on (Figure, A; FLS, fluoroscopy LS; FL, fluoroscopy left). To label the points of the left and right PVs, we used $45^{\circ}$ left anterior oblique projection and $30^{\circ}$ right anterior oblique projection of fluoroscopy, respectively. All marked points were stored as "map-1." The operator was blinded to the reconstructed CT image before and during the processing. These fluoroscopy planes were used to represent the encircled ablation performed without the guidance of CARTO-Merge.

Image integration: Next, the 3D electroanatomic map of the LA was reconstructed by a mapping catheter under the guidance of the CARTO system by using a point-by-point method. The initial registration process on the CARTO workstation involved identifying specific anatomic locations

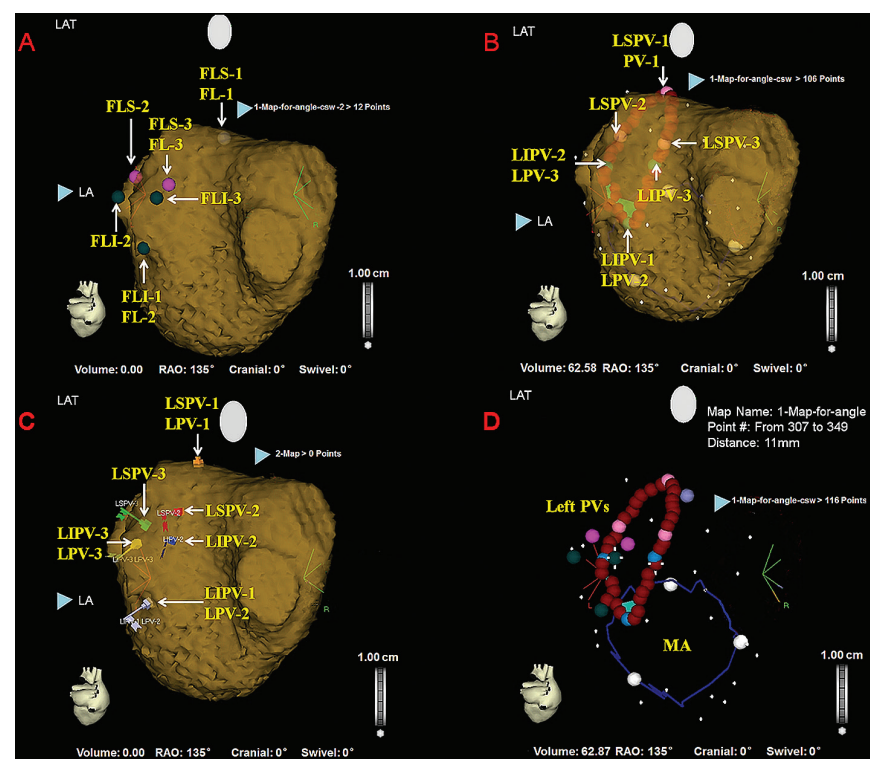

A representative diagram to show the fluoroscopy planes, ablation planes, and standard planes. (A), Predicted planes navigated by using standard angiography guidance, without CARTO-Merge (fluoroscopy planes); (B), ablation planes navigated by CARTO-Merge; (C), standard panels navigated by using the CT image (this step was performed without viewing the actual ablation rings); (D), some PV planes defined by the method without CARTO-Merge guidance had notable errors (about $11 \mathrm{~mm}$ inside the correct location). We found that the ring of actual ablation locations (the colorful ring, also shown in panel B) was very similar to the standard locations defined by CT image (locations also shown in panel $\mathrm{C}$ ), but was significantly different to predicted locations defined without CARTO-Merge guidance (also shown in panel A). PV, pulmonary vein; LS, left superior; LI, left inferior; FLS, fluoroscopy LS; FLI, fluoroscopy LI; FL, fluoroscopy left; LSPV, LS PV; LIPV, LI PV; LPV, left ipsilateral PV; MA, mitral annulus; LA, left atrium.

(landmarks) in the posterior-inferior junction of the RIPVLA, which was identified by withdrawing the mapping catheter from the RIPV to the LA. The imported CT image was locked at this point and registered with the electroanatomic map, and further refinement of the imported image overlapping the map was obtained by the "points to surface" phase. In this phase, endocardial points were collected to cover all the areas of the endocardial surface of the LA; we set the rejecting points with a $>5-\mathrm{mm}$ difference between the CT image and the map. Integration accuracy was evaluated by navigating the mapping catheter throughout the PV ostia identified by PV angiography, fluoroscopy, the CARTO system, and the mapping catheter. $\mathrm{PV}$ isolation was performed under the guidance of CARTO-Merge, the 3D electroanatomic LA map, and fluoroscopy. After circumferential PV isolation was completed, the reconstructed LA map (with ablation rings) was restored as "map-2" for offline analysis before other ablations were performed to complete the procedures on the LA map.

\section{Definition of the Ablation Plane and the Image Plane}

In the offline analysis, 3 ablation points were defined and labelled from the anterior, and posterior and superior or inferior segments of the actual ablation rings were employed to 
Table 1. Predicted Distances (from Fluoroscopy Planes to Standard Image Planes) and Actual Distances (from Ablation Planes to Standard Image Planes)*

\begin{tabular}{llll}
\hline PV Planes & $\begin{array}{c}\text { Predicted Distance } \\
\text { (Not Navigated by } \\
\text { CARTO-Merge) }\end{array}$ & $\begin{array}{c}\text { Actual Distance } \\
\text { (Navigated by } \\
\text { CARTO-Merge) }\end{array}$ & $P$ \\
\hline LIPV & $2.60 \pm 2.95 \mathrm{~mm}$ & $2.98 \pm 1.78 \mathrm{~mm}$ & .458 \\
LSPV & $2.48 \pm 1.84 \mathrm{~mm}$ & $1.86 \pm 1.25 \mathrm{~mm}$ & .066 \\
LPV & $2.89 \pm 2.49 \mathrm{~mm}$ & $1.36 \pm 1.27 \mathrm{~mm}$ & $<.001 \dagger$ \\
RIPV & $3.51 \pm 2.46 \mathrm{~mm}$ & $2.58 \pm 1.69 \mathrm{~mm}$ & $.034 \dagger$ \\
RSPV & $3.47 \pm 2.97 \mathrm{~mm}$ & $2.10 \pm 1.80 \mathrm{~mm}$ & $.009 \dagger$ \\
RPV & $4.13 \pm 3.47 \mathrm{~mm}$ & $2.33 \pm 1.71 \mathrm{~mm}$ & $.002 \dagger$ \\
\hline
\end{tabular}

*Where PV indicates the pulmonary vein, the abbreviations are as follows: LIPV, left inferior PV; LSPV, left superior PV; LPV, left ipsilateral PV; RIPV, right inferior PV; RSPV, right superior PV; and RPV, right ipsilateral PV. $\dagger P<.05$.

represent the actual ablation planes of the 4 separate PVs in map-2. The ablation planes of the ipsilateral PVs were also identified by the superior, inferior, and anterior or posterior ablation points of the actual ablation rings in map-2. In this procedure, the operator was blinded to integrated map of the CT image and the fluoroscopy planes. Points were marked in the CARTO system as LSPV-1 to LSPV-3, LPV-1 to LPV-3, and so on (Figure, B; LPV, left ipsilateral PV).

Three landmarks, at the anterior, posterior, and superior or inferior walls of the 4 separate PVs, were labelled at the PV-LA junction of the reconstructed CT image (created with the CARTO system) to represent the standard PV plane. The standard planes of the ipsilateral PVs were also identified by the superior, inferior, and anterior or posterior landmarks of the ipsilateral PVs. Those landmarks were named LSPV-1 to LSPV-3, LPV-1 to LPV-3, and so on (Figure, C). This process was performed by 3 experienced observers who were blinded to the actual ablation rings and the fluoroscopy planes map, but the operator was not blinded [Chen 2013].

All data, including the fluoroscopy planes, ablation planes, standard planes, CT images, and registration files for each case, were stored in a single file for angle and distance analysis in the CARTO system.

\section{Comparison between Fluoroscopy, Ablation, and Image Planes}

Two sets of indexes were investigated in the present study. One index was the conventional distance between planes [Kimura 2013]. Another index was the angle between planes, based on our previous study [Chen 2013]. We defined the predicted angle (distance) as the angle (distance) between the standard planes and the fluoroscopy planes, whereas the actual angle (distance) was defined as the angle (distance) between the standard planes and the actual ablation planes. Full details of the methods for calculating the angle and distance are
Table 2. Predicted Angles (from Fluoroscopy Planes to Standard Image Planes) and Actual Angles (from Ablation Planes to Standard Image Planes)

\begin{tabular}{lccc}
\hline PV Planes & $\begin{array}{c}\text { Predicted Angles } \\
\text { (Not Navigated by } \\
\text { CARTO-Merge) }\end{array}$ & $\begin{array}{c}\text { Actual Angles } \\
\text { (Navigated by } \\
\text { CARTO-Merge) }\end{array}$ & $P$ \\
\hline LIPV & $20.69^{\circ} \pm 13.91^{\circ}$ & $15.80^{\circ} \pm 7.64^{\circ}$ & $.040^{*}$ \\
LSPV & $23.21^{\circ} \pm 12.97^{\circ}$ & $15.17^{\circ} \pm 7.20^{\circ}$ & $.001^{*}$ \\
LPV & $30.52^{\circ} \pm 20.97^{\circ}$ & $13.91^{\circ} \pm 6.20^{\circ}$ & $<.001^{*}$ \\
RIPV & $17.94^{\circ} \pm 8.89^{\circ}$ & $13.58^{\circ} \pm 8.01^{\circ}$ & $.014^{*}$ \\
RSPV & $33.38^{\circ} \pm 15.86^{\circ}$ & $11.20^{\circ} \pm 6.75^{\circ}$ & $<.001^{*}$ \\
RPV & $26.40^{\circ} \pm 18.89^{\circ}$ & $13.27^{\circ} \pm 6.14^{\circ}$ & $<.001^{*}$ \\
\hline
\end{tabular}

$* P<.05$.

described in our previous study [Chen 2013]. Briefly, the angle between 2 correlative planes was computed as the dihedral angle of the 2 planes. If these planes were coplanar, the angle between them was regarded as $0^{\circ}$. If not coplanar, the angle was presented as an acute angle. The distance between 2 corresponding planes was calculated as the distance between the 2 geometric centers of the 2 planes, defined by every 3 points.

Follow-up: After the finishing of the procedures, all patients were required to undergo periodic follow-up (at least once a month for 1 year and then every 6 months) in an outpatient clinic. The presence or absence of $\mathrm{AF}$ was evaluated by symptoms, electrocardiogram recordings, and 24-hour ambulatory monitoring. Follow-up monitoring was performed at 1, 3, 6, and 12 months after the operation, and once every 6 months thereafter. AF recurrence was defined as any $\mathrm{AF}$ episode lasting greater than 30 seconds on 24-hour ambulatory monitoring or electrocardiography. Freedom from AF recurrence was defined as no $\mathrm{AF}$ occurrence during medication stoppage after a 3-month blanking period.

\section{Statistical Analysis}

Values are recorded as the mean \pm standard deviation or as percentages (for categorical variables), unless otherwise indicated. All statistical analyses were performed by using IBM SPSS Statistics for Windows, Version 19.0 (IBM Corp., Armonk, NY, USA; released 2010). Categorical variables were compared with $\chi^{2}$ or Fisher tests, and continuous variables were compared with unpaired Student $t$ tests. All tests were 2-tailed, and statistical significance was defined as a $\mathrm{P}$ value less than .05 .

\section{RESULTS}

\section{Clinical Characteristics of the Participants}

A total of 48 consecutive patients (average age $57.7 \pm 11.5$ years; $64.6 \%$ males, 29 with paroxysmal $\mathrm{AF}$ ) with typical $\mathrm{PV}$ branching patterns were enrolled in the present study. The average duration of $\mathrm{AF}$ was $6.5 \pm 7.3$ years, and the average 
LA diameter was $43.6 \pm 6.2 \mathrm{~mm}$. There were 22 patients with comorbidities, including hypertension (20 cases), diabetes mellitus (3 cases), ischemic stroke ( 2 cases), transient ischemic attack (1 case), and hypertrophic cardiomyopathy (1 case). Cardiological history was positive for paroxysmal supraventricular tachycardia in 2 cases, and for complete left bundle branch block, complete right bundle branch block, and permanent pacemaker implantation for sick sinus syndrome in one case each.

A mean of $77.7 \pm 19.2$ endocardial points were sampled for surface registration. The average surface-to-point distance was $1.88 \pm 0.32 \mathrm{~mm}$ (range $1.20-2.60 \mathrm{~mm}$ ).

\section{Ablation Navigated by CARTO-Merge Significantly Improved Intraoperative Accuracy}

Ablation navigated by CARTO-Merge significantly reduced the actual distance (from image to ablation planes) in the LPV $(P<.001)$, RIPV $(P<.05)$, right ipsilateral PV (RPV, $P<.01)$, and RSPV $(P<.01)$, but not in LIPV $(P=.458)$ and LSPV $(P=0.066)$, in comparison with the predicted distance (from image planes to fluoroscopy planes) (Table 1).

Concerning the actual angle (between image and ablation planes), ablation navigated by CARTO-Merge significantly reduced the angles in all PV planes $(P<.05$; Table 2$)$ when compared with the predicted distance (between image and fluoroscopy planes). Overall, the angle exhibited better sensitivity because angles in all PV planes were significantly reduced, although not all distances in PV planes were significantly reduced.

Given that navigation by CARTO-Merge can reduce the distance and angle when comparing actual with predicted data, the accuracy of the ablation was markedly improved compared to those without image integration. In the representative sample of this study, the PV planes defined by CARTOMerge (Figure, B) were very similar to those defined by CT image (Figure, C), whereas the regular angiography methods could show incorrect locations (Figure, D) that were different to those defined by CT imaging (Figure, A).

\section{Clinical Outcomes}

There were 2 complications (one femoral hematoma and one femoral pseudoaneurysm), but these were treated conventionally without any long-term sequelae. No pericardial effusion or PV stenosis was encountered. After the first procedure, 17 patients $(35.4 \%)$ suffered from recurrent atrial arrhythmias, including 12 with $\mathrm{AF}$ and 5 with atrial flutter or atrial tachycardia; another 4 (8.3\%) underwent a second ablation for recurrent atrial arrhythmias. During a mean followup of $44.2 \pm 4.1$ months after the last procedure, 35 of the 48 patients $(72.9 \%)$, including 23 paroxysmal AF, were free of $\mathrm{AF}$ recurrence and remained in normal sinus rhythm.

\section{DISCUSSION}

The present study indicates that not only the conventional distance between ablation planes and standard planes but also the angle between ablation planes and standard planes were significantly reduced by image integration. These reductions were associated with marked improvements in ablation accuracy. To the best of our knowledge, this is the first quantitative evaluation of the angles between planes during ablation for AF. We conclude that both the distance and the angle should be employed as indexes of ablation accuracy and argue that using image integration with both indexes significantly improves accuracy when performing ablation of AF.

In this study, we evaluated a new index that compares the difference between the actual and predicted angles, based on our previous work. In that earlier research, we showed that significant torsion variance existed between the ipsilateral PVs [Chen 2013] and that intraoperatively, this torsion angle should be considered along with the existing distance index to improve the accuracy of ablation. It was anticipated that this approach would not only reduce the risk of several complications but also the surgical time of PV isolation.

To assess our hypotheses, we compared 2 angles in the present study: (a) the predicted angle, which is the angle between the predicted plane defined by PV angiography and the standard PV plane; and (b) the actual angle, which is the angle between actual ablation planes, as performed on the merged image, and the standard PV plane. Smaller actual angles indicate better accuracy during the ablation processes, and our data show that the angles in all PV planes were significantly reduced with guidance by merging images, compared with guidance by $\mathrm{PV}$ angiography alone $(P<.05$; Table 2$)$. Regarding the conventional distance index, we found that only LPV, RIPV, RDPV, and RPV exhibited significant differences, but that LIPV and LSPV did not $(P>.05$; Table 1$)$. Thus, we conclude that the new angle is a more sensitive index than the conventional distance index.

We know that effective and safe PV isolation is crucial during ablation. The operator must clearly understand the PV-LA anatomy to perform precise operations at this location. The use of appropriate tools is indispensable for identifying the PV ostia, and perhaps more importantly, the realtime relative location of the ablation catheter. Understanding the distance alone, however, is insufficient, and consideration of the torsion angle can help the operator form a better 3D anatomical understanding. The present study provides evidence for the potential clinical usefulness of the torsion angle as an index of ablation accuracy. We propose that the angle is a sensitive index that could improve ablation accuracy and that its use should be considered in future operations.

Concerning the conventional distance index, many previous studies have reported that image integration technology can significantly reduce the average distance of mapping points. Kimura et al, for example, reported that the distance was reduced to $1.08 \pm 0.14 \mathrm{~mm}$ by image integration [Kimura 2013]. In the present study, in which $77.7 \pm 19.2$ endocardial points were sampled for surface registration, we showed a mean distance of $1.88 \pm 0.32 \mathrm{~mm}$ (range $1.20-2.60 \mathrm{~mm}$ ). Therefore, image integration (or registration) accuracy in the present study was acceptable.

Along with highlighting the benefits of the new angle as an index of ablation accuracy, we have provided evidence for the benefit of image integration in ablation processes. Compared with conventional 2D methods like PV angiography and intracardiac echocardiography, 3D imaging by CT or 
MRI is better able to provide anatomical localization. Using $3 \mathrm{D}$ imaging during ablation is not a new technology [Dong 2006]. Indeed, integrating 3D mapping systems with CT images has facilitated anatomy-based radiofrequency catheter ablation for AF, by direct visualization of the PVs (including the number and the details of any special anatomical features, such as common ostia, early branching, or torsion) and individual LA geometry [Martinek 2007]. It is helpful for the operator to understand the 3D locations of the PV ostia, the branching and supernumerary PVs, and the catheter. Moreover, it can aid the operator to understand torsion between ipsilateral PVs, thereby helping him or her to avoid singleplane circumferential ablation [Chen 2013]. Successful integration of this technique may contribute to greater accuracy in lesion ablation and catheter manipulation around the PV. The present study provides stronger evidence of the benefits of the 3D image integration although many laboratories do not use image integration for radiofrequency catheter ablation. Some researchers performed PV isolation by local electrogram. However, our results in the present study exhibited that $3 \mathrm{D}$ image integration can improve not only the distance, but also the angle, that is good for precisely understanding the PV anatomic location for performing better ablation.

Finally, we showed that clinical outcomes were improved by the greater accuracy in the operation. Although in the experimental design of the present study we cannot compare the outcomes between patients with and without 3D image integration, we can compare the outcomes with the previous analogous studies. Many previous studies have documented better outcomes with image integration [Bertaglia 2009, Kimura 2013]. In a recent study, recurrence of atrial tachyarrhythmia was reduced by catheter ablation for AF, with $73 \%$ of patients (with image integration) free of atrial tachyarrhythmia after a 24-month follow-up time [Marai 2016]. These data are very close to our findings in this study $(72.9 \%$, during $44.2 \pm 4.1$ months follow-up time), whereas the rate of being free of atrial tachyarrhythmia in patients without image integration was only 55\% [Marai 2016]. By comparing the outcomes in previous analogous study, our results also exhibit a better outcome from the use of the $3 \mathrm{D}$ image integration.

\section{CONCLUSIONS}

Taken together, the present study shows that navigation by image integration can help reduce both the angle and distance between ablation lesions and the actual PV-LA junction. The resulting improvements in ablation accuracy may be associated with better clinical outcomes, but this will need to be verified by further study. In the meantime, we believe our results justify using the angle in combination with the conventional distance index to provide a more sensitive index of ablation accuracy.

\section{REFERENCES}

Bertaglia E, Della Bella P, Tondo C, et al. 2009. Image integration increases efficacy of paroxysmal atrial fibrillation catheter ablation: results from the CartoMerge ${ }^{\mathrm{TM}}$ Italian Registry. Europace 11:1004-10.

Calkins H, Kuck KH, Cappato R, et al. 2012. 2012 HRS/EHRA/ECAS expert consensus statement on catheter and surgical ablation of atrial fibrillation: recommendations for patient selection, procedural techniques, patient management and follow-up, definitions, endpoints, and research trial design. J Interv Card Electrophysiol 33:171-257.

Chen S, Meng W, He DS, et al. 2012. Blocking the pulmonary vein to left atrium conduction in addition to the entrance block enhances clinical efficacy in atrial fibrillation ablation. Pacing Clin Electrophysiol 35:524-31.

Chen S, Lu X, Zhen Y, et al. 2013. Spatial torsion of the ipsilateral superior and inferior pulmonary veins. J Interv Card Electrophysiol 37:35-40.

Desjardins B. 2016. Imaging for cardiac electrophysiology. SA J Radiol 20:a1048.

Dong J, Dickfeld T, Dalal D, et al. 2006. Initial experience in the use of integrated electroanatomic mapping with three-dimensional MR/CT images to guide catheter ablation of atrial fibrillation. J Cardiovasc Electrophysiol 17: 459-66.

Kimura M, Sasaki S, Owada S, et al. 2013. Validation of accuracy of three-dimensional left atrial CartoSound ${ }^{\mathrm{TM}}$ and CT image integration: influence of respiratory phase and cardiac cycle. J Cardiovasc Electrophysiol 24:1002-7.

Kistler PM, Rajappan K, Jahngir M, et al. 2006. The impact of CT image integration into an electroanatomic mapping system on clinical outcomes of catheter ablation of atrial fibrillation. J Cardiovasc Electrophysiol 17:1093-101.

Mansour M, Holmvang G, Sosnovik D, et al. 2004. Assessment of pulmonary vein anatomic variability by magnetic resonance imaging. J Cardiovasc Electrophysiol 15: 387-93.

Marai I, Suleiman M, Blich M, Lessick J, Abadi S, Boulos M. 2016. Impact of computed tomography image and contact force technology on catheter ablation for atrial fibrillation. World J Cardiol 8:317-22.

Martinek M, Nesser HJ, Aichinger J, Boehm G, Purerfellner H. 2007. Impact of integration of multislice computed tomography imaging into three-dimensional electroanatomic mapping on clinical outcomes, safety, and efficacy using radiofrequency ablation for atrial fibrillation. Pacing Clin Electrophysiol 30: 1215-23.

Shigenaga Y, Okajima K, Ikeuchi K, et al. 2016. Usefulness of non-contrast-enhanced MRI with two-dimensional balanced steady-state free precession for the acquisition of the pulmonary venous and left atrial anatomy pre catheter ablation of atrial fibrillation: Comparison with contrast enhanced CT in clinical cases. J Magn Reson Imaging 43:495-503.

Tops LF, Bax JJ, Zeppenfeld K, et al. 2005. Fusion of multislice computed tomography imaging with three-dimensional electroanatomic mapping to guide radiofrequency catheter ablation procedures. Heart Rhythm 2:1076-81. 